\title{
CREB in the Mouse SCN: A Molecular Interface Coding the Phase- Adjusting Stimuli Light, Glutamate, PACAP, and Melatonin for Clockwork Access
}

\author{
Charlotte von Gall, ${ }^{1}$ Giles E. Duffield, ${ }^{2}$ Michael H. Hastings, ${ }^{2}$ Michael D. A. Kopp, ${ }^{1,3}$ Faramarz Dehghani, ${ }^{1}$ \\ Horst-Werner Korf, ${ }^{1}$ J örg H. Stehle ${ }^{1}$ \\ ${ }^{1 D}$. Senckenbergische Anatomie, Anatomisches Institut II, J ohann Wolfgang Goethe-Universität, D-60590 Frankfurt, \\ Germany, 2Department of Anatomy, University of Cambridge, Cambridge CB2 3DY, United Kingdom, and \\ 3Max-Planck-Institut für Physiologische und Klinische Forschung, W. G. Kerckhoff-Institut, \\ D-61231 Bad Nauheim, Germany
}

The suprachiasmatic nucleus (SCN) is a central pacemaker in mammals, driving many endogenous circadian rhythms. An important pacemaker target is the regulation of a hormonal message for darkness, the circadian rhythm in melatonin synthesis. The endogenous clock within the SCN is synchronized to environmental light/dark cycles by photic information conveyed via the retinohypothalamic tract (RHT) and by the nocturnal melatonin signal that acts within a feedback loop. We investigated how melatonin intersects with the temporally gated resetting actions of two RHT transmitters, pituitary adenylate cyclase-activating polypeptide (PACAP) and glutamate. We analyzed immunocytochemically the inducible phosphorylation of the transcription factor $\mathrm{Ca}^{2+} / \mathrm{CAMP}$ response elementbinding protein (CREB) in the $\mathrm{SCN}$ of a melatonin-proficient $(\mathrm{C} 3 \mathrm{H})$ and a melatonin-deficient (C57BL) mouse strain. In vivo, light-induced phase shifts in locomotor activity were consistently accompanied by CREB phosphorylation in the SCN of both strains. However, in the middle of subjective nighttime, light induced larger phase delays in C57B L than in $\mathrm{C} 3 \mathrm{H}$ mice. In vitro, PACAP and glutamate induced CREB phosphorylation in the SCN of both mouse strains, with PACAP being more effective during late subjective daytime and glutamate being more effective during subjective nighttime. Melatonin suppressed PACAP - but not glutamate-induced phosphorylation of CREB. The distinct temporal domains during which glutamate and PACAP induce CREB phosphorylation imply that during the light/dark transition the SCN switches sensitivity between these two RHT transmitters. Because these temporal domains are not different between $\mathrm{C} 3 \mathrm{H}$ and $\mathrm{C} 57 \mathrm{BL}$ mice, the sensitivity windows are set independently of the rhythmic melatonin signal.

Key words: suprachiasmatic nucleus; circadian; phase shifts; mice; brain slice; CREB $\left(\mathrm{Ca}^{2+} / \mathrm{CAMP}\right.$ response elementbinding protein); glutamate; PACAP (pituitary adenylate cyclase-activating polypeptide); melatonin
$M$ any biological rhythms persist in the absence of environmental cues (zeitgebers) with a circadian period of $\sim 24 \mathrm{hr}$. In mammals, important circadian rhythms are driven by an oscillator in the hypothalamic suprachiasmatic nucleus (SC N) (K lein et al., 1991). The principal cue entraining the endogenous rhythm in SCN activity to ambient light/dark conditions is photic information conveyed by the retinohypothalamic tract ( $\mathrm{RHT}$ ).

$M$ echanisms of cellular processing required to integrate environmental stimuli into the endogenous genetic program of circadian timing in the SC $\mathrm{N}$ to pace biological rhythms and anticipate cyclic changes in the light/dark regimen are not known. The sensitivity of the SC N to the resetting actions of neurochemical factors implicated in entrainment is restricted to discrete temporal windows. These windows, overlapping with equally discrete molecular gates for distinct signaling pathways, are notably out of

\footnotetext{
Received J uly 29, 1998; revised Sept. 28, 1998; accepted O ct. 2, 1998.

This study was supported by grants from the D eutsche Forschungsgemeinschaft (H.-W.K. and J.H.S.), the Wellcome Trust (G.E.D. and M .H.H.), and the E uropean Federation of Experimental Morphology (C.v.G.). We thank H. Wicht for advice with image analysis, H. M eissl for helpful discussion, and I. Schneider-H üther for technical support.

Parts of this paper have been presented at the $1998 \mathrm{M}$ eeting of the A natomical Society, Greifswald, Germany, and appeared in the meeting proceedings.

Correspondence should be addressed to Dr. Jörg H. Stehle, Dr. Senckenbergische A natomie, A natomisches Institut II, Johann Wolfgang Goethe-U niversität, Theodor-Stern-K ai 7, D-60590 Frankfurt, G ermany.

Copyright (c) 1998 Society for Neuroscience 0270-6474/98/1810389-09\$05.00/0
}

phase with each other. During daytime, phase shifts can be induced by activation of the CA M P-signaling pathway (Prosser and Gillette, 1989), whereas the prevailing stimulus for phase shifts during nighttime results from activation of the $\mathrm{Ca}^{2+}$. and/or the CG M P-signaling pathway(s) (Prosser et al., 1989; Ding et al., 1998). In the rodent R H T, glutamate signals "light" to the pacemaker during nighttime ( $C$ astel et al., 1993; D ing et al., 1994, 1997). Conversely, the pituitary adenylate cyclase-activating polypeptide (PACAP) makes the SC N sense "darkness" during daytime, by elevating the intracellular CAMP concentration (Hannibal et al., 1997; K opp et al., 1997). At dusk and possibly also at dawn, a sensitivity window exists for the pineal hormone melatonin to affect clock activity (R edman et al., 1983; C assone et al., 1987; Stehle et al., 1989; McA rthur et al., 1991). Thus, melatonin, the hormonal message for darkness, may fine-tune circadian timing via interference with other pathways, thereby defining SC $N$ sensitivity to resetting cues. Because phase shifts in SC N activity require protein synthesis (Z hang et al., 1996) and induce D N A -binding proteins (K ornhauser et al., 1990; R usak et al., 1990; G inty et al., 1993; Stehle et al., 1996), transcription is part of pacemaker adjustment. Therefore, a molecular interface for neuronal (R H T neurotransmission) and/or endocrine (melatonin) cues must be able to serve different signaling pathways, to react fast, and to convey external stimuli rapidly to the transcriptional machinery necessary for consolidation or even initiation of 
phase shifts. There is increasing evidence that the transcription factor $\mathrm{Ca}^{2+} / \mathrm{CA}$ M P response element-binding protein (CREB) may act as an integrator involved in resetting circadian rhythms (G inty et al., 1993; M cNulty et al., 1998).

To gain further insights into the temporal gating of the SC N to resetting cues and to examine the potential impact of melatonin, we compared oscillator properties of $\mathrm{C} 3 \mathrm{H}$ mice with a rhythmic melatonin synthesis, with those of C57BL mice with an undetectable melatonin synthesis in vivo (E bihara et al., 1986; G oto et al., 1989). U sing both in vivo and in vitro approaches, we found that phase-shifting stimuli converge within the $\mathrm{SCN}$ at a molecular level with the phosphorylation of CREB. Pacemaker-resetting cues act on CREB at different temporal sensitivity windows that are set intrinsically, independently of the endogenous melatonin signal.

\section{MATERIALS AND METHODS}

A nimals and in vivo studies. A II animal experimentation reported in this manuscript was conducted in accordance with the Policy on the $U$ se of A nimals in N euroscience R esearch and the Policy on E thics as approved by the Society for Neuroscience. Male $\mathrm{C} 3 \mathrm{H}$ (substrains $\mathrm{HeN}$ or $\mathrm{J}$ ) and C57BL / 6 mice aged 6-10 weeks (Charles River Wiga, Sulzfeld, Germany) were housed in individual cages equipped with running wheels and with constant room temperature and food and water available ad libitum. Because no differences between the two $\mathrm{C} 3 \mathrm{H}$ substrains were evident in any of the experiments conducted, these animals are further referred to as $\mathrm{C} 3 \mathrm{H}$ mice. Wheel-running activity was recorded continuously and analyzed by a computer system (Viglen Contender PC, A lberton, UK) running Dataquest IV software (D ata Sciences, Frankfurt, Germany). The mice were entrained to a photoperiod of $12 \mathrm{hr}$ of light ( $250 \mathrm{lux}$ ):12 hr of darkness (dim red light $<15$ lux) for at least 1 week before the experiments. Subsequently, animals were transferred into constant dim red light (DD), and free-running activity rhythms were monitored for $16 \mathrm{~d}$ consecutively. A ctivity onset was defined as circadian time 12 (CT12). The light pulses were delivered by moving the animals from the DD environment to a monochromatic light source with an intensity of 1000 lux (15 $\mathrm{min}$ ) in a neighboring room during the projected subjective day (CT06 and CT10) or subjective night (CT14 and CT18). Control experiments were conducted by moving the mice without light exposure. To examine resetting responses, we exposed animals to between one and three light pulses at different circadian times, with at least $5 \mathrm{~d}$ between pulses to allow for consolidation of phase shifts. Phase shifts were determined by measuring the phase difference between eye-fitted lines through successive daily activity onsets, using observers blind to treatment. N egative values represent stimulus-induced phase delays; positive values represent phase advances.

To investigate in vivo the light-induced phosphorylation of CREB in the SCN, we anesthetized animals after light or sham exposure with sodium pentobarbital ( $1.6 \mathrm{gm} / \mathrm{kg}$ of body weight, i.p.) and transcardially perfused the animals with $4 \%$ paraformaldehyde (PFA) in PBS with heparin (2000 U/mouse) as an additive. Brains were removed, post-fixed for $2 \mathrm{hr}$ in $4 \%$ PFA, and then cryoprotected with 20\% sucrose in PBS overnight. Coronal hypothalamic sections ( $40 \mu \mathrm{m}$ for immunocytochemistry; $100 \mu \mathrm{m}$ for confocal laser microscopy) were cut on a freezing microtome and collected into PBS for immediate free-floating immunocytochemistry (see below).

In vitro studies. M ice kept under standard 12:12 hr light/dark conditions were decapitated, brains were removed quickly, and coronal hypothalamic slices ( $400 \mu \mathrm{m}$ thick) containing the paired SC N were cut at $4^{\circ} \mathrm{C}$ using a vibratome. To avoid phase shifting of the circadian clock by brain slicing (Gillette, 1986), we prepared slices at least $2 \mathrm{hr}$ before the onset of the dark phase. Stimulations of slices were conducted subsequently at zeitgeber time 06 (ZT06), ZT10, ZT14, and ZT18, with ZT12 designated as the onset of the donor's dark phase. A fter preincubation for at least $2 \mathrm{hr}$ in artificial CSF [aC SF (in mM), $\mathrm{NaCl} 145 ; \mathrm{KCI} 5 ; \mathrm{C} \mathrm{aCl}_{2}$ 1.8; $\mathrm{M} \mathrm{gCl}_{2}$ 0.8; HEPES 10; and glucose 10; $\mathrm{pH} \mathrm{7.35;} \mathrm{at} 37^{\circ} \mathrm{C}$ ], slices were stimulated with glutamate [100 $\mu \mathrm{M}$; a dose according to Shirakawa and M oore (1994)] or PA CA P [100 nM; a dose according to $\mathrm{H}$ annibal et al. (1997)] for $15 \mathrm{~min}$. To investigate the impact of melatonin on PA CA Pergic and glutamatergic effects, we added melatonin [1 nM; a dose according to M CNulty et al. (1994)] alone for $30 \mathrm{~min}$ to aC SF or for $15 \mathrm{~min}$ before and during glutamate or PA CA P application. U nstimulated slices served as controls. Subsequently, slices were fixed with 4\% PFA for 12-16 $\mathrm{hr}$, cryoprotected (20\% sucrose in PBS), and sectioned on a cryostat. Sections $(14 \mu \mathrm{m})$ were mounted on gelatin-coated slides and stored at $-20^{\circ} \mathrm{C}$ until immunocytochemistry was performed.

Immunocytochemistry. I mmunoreaction (IR) for the phosphorylated form of CREB (PCREB) in the SCN was visualized with a standard avidin-biotin labeling method, with diaminobenzidine as the chromogen as described (Sumova et al., 1994; Tamotsu et al., 1995). Primary polyclonal antibodies against CREB, phosphorylated at the residue $\mathrm{Ser}^{133}$ ( $p C R E B ;$ N ew England Biolabs, Beverly, M A ; U pstate Biotechnology, Lake Placid, NY), were used at dilutions of 1:1000 and 1:500, respectively.

For double-label immunofluorescence, free-floating sections were incubated with the rabbit anti-pCREB antibody (1:100; New England Biolabs) and a monoclonal anti-V I P antibody (1:200; Bio Trend, Köln, Germany). Subsequently, the sections were incubated for $1 \mathrm{hr}$ at room temperature in a mixture of goat anti-rabbit C y3 (1:500; J ackson I mmunoR esearch, West Grove, PA) and goat anti-mouse fluorescein isothiocyanate (FITC; 1:500; Sigma, St. L ouis, M O). The buffer for all incubation steps was PBS with $1 \%$ BSA and $0.5 \%$ Triton $X-100$; intermediate washing steps were done in PBS. Sections were mounted on gelatincoated slides and coverslipped with fluorescent mounting medium ( $D$ ako, Carpinteria, CA).

Confocal laser microscopy. A Z eiss L SM 510 confocal imaging system equipped with a monochromatic argon laser light source (wavelength, 458 and $488 \mathrm{~nm}$ ) was attached to an inverse A xiovert $100 \mathrm{Z}$ eiss (G öttingen, Germany) microscope. The $488 \mathrm{~nm}$ line of this laser was used to excite the FITC fluorophore, the beam passing through a dichroic beam splitter (F T 488) and an emission bandpass filter (BP 505-530). A second helium-neon laser was used to emit monochromatic light at $543 \mathrm{~nm}$. This laser line was applied to excite the $\mathrm{C}$ y3 fluorophore, using a dichroic beam splitter FT 543 and an emission bandpass filter BP 580-615. I mmunofluorescence images of both channels were stored for further analysis as digitized images with an eight bit resolution $(1024 \times 1024$ pixels).

Analysis of $P C R E B-I R$. Initially, all sections of the SCN immunostained for $P C R E B$ were inspected routinely with a $Z$ eiss microscope (A xioplan; $100 \times$ ) equipped with a video camera that was connected to a computerized image analysis system (VIDAS, Kontron, Germany). Semiquantitative analyses were performed as described (Pfeffer et al., 1998). Briefly, the images were digitized; background staining was used to define the lower threshold. Within the area of the SC N all cell nuclei showing a pCREB-IR exceeding the threshold were marked. Because pCREB-stained cell nuclei were homogeneously distributed throughout the rostrocaudal extent of the SCN, three sections of the intermediate aspect of the SCN were chosen at random for further analysis. Nuclei with a PCREB-IR were counted in a blind manner, and the mean numbers ( \pm SEM ) of stained nuclei within SC N boundaries were used for statistical analysis.

Statistical analysis. Statistical analysis of the in vivo and the in vitro experiments was performed using Graph Pad Prism (Graph Pad, San Diego, CA). Significant differences between groups were determined with a one-way A NOVA, followed by Tukey's post hoc test. Data are presented as the mean \pm SEM. Values were considered significantly different with $p<0.05$.

Materials. D rugs and chemicals were obtained from the following sources: PA CA P, melatonin, and forskolin were from $\mathrm{C}$ albiochem ( $\mathrm{L} \mathrm{U}$ cerne, Switzerland); all others drugs and chemicals were purchased from Sigma.

\section{RESULTS}

\section{Effects of light on locomotor activity in mice kept under DD}

The period length of the locomotor activity rhythm in constant darkness was not significantly different between C57BL (23.77 \pm $0.11 \mathrm{hr} ; \mathrm{n}=16)$ and $\mathrm{C} 3 \mathrm{H}(23.65 \pm 0.07 \mathrm{hr} ; \mathrm{n}=16)$ mice. E xposure to a light pulse during subjective day (CT06, for $\mathrm{C} 3 \mathrm{H}$, $\mathrm{n}=9$; for $\mathrm{C} 57 \mathrm{BL}, \mathrm{n}=6$; C T 10, for $\mathrm{C} 3 \mathrm{H}, \mathrm{n}=5$; for $\mathrm{C} 57 \mathrm{BL}, \mathrm{n}=$ 6) or sham exposure in $D D$ (controls, for $\mathrm{C} 3 \mathrm{H}, \mathrm{n}=10$; for C57BL, $n=8$ ) had no significant effects on the phase of locomotor activity rhythms (Figs. 1, 2A). Light pulses applied during subjective night ( C T 14 and C T 18) resulted in stable phase delays 


\section{$\mathrm{C} 3 \mathrm{H}$}

A

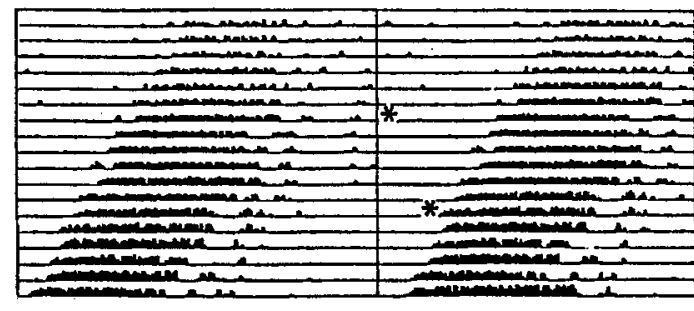

C

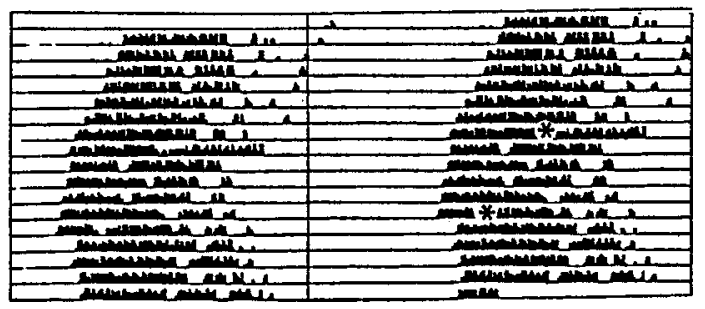

C57BL

B

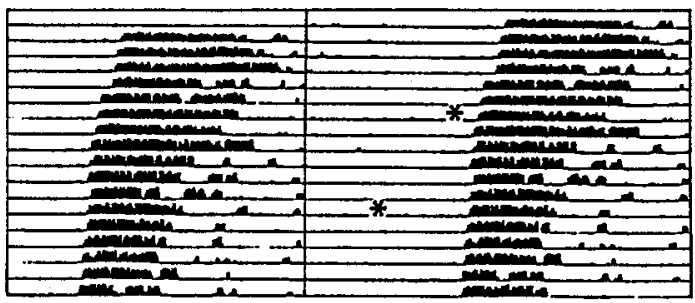

D

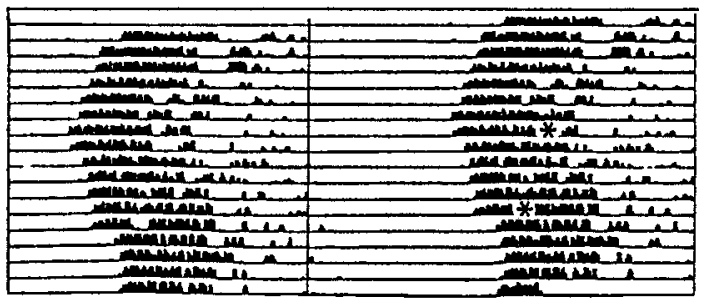

Figure 1. Light-induced effects on locomotor activity of $\mathrm{C} 3 \mathrm{H}$ and $\mathrm{C} 57 \mathrm{BL}$ mice. A nimals were kept under constant darkness (dim red light). R epresentative double-plotted actograms of animal wheel-running activity are shown with asterisks indicating the time of light pulses ( 15 min; 1000 lux). Light presented during subjective day (C T06 and C T 10) had little or no effect in C $3 \mathrm{H}$ ( A) and C57B L (B) mice, whereas light applied during subjective night (CT14 and CT18) resulted in stable phase delays in C3H (C) and C57BL (D) mice.

in both strains of mice (Figs. 1, 2A); light exposure of animals 2 $\mathrm{hr}$ after activity onset (CT14) was most effective to induce phase delays in C57BL $(-1.78 \pm 0.18 \mathrm{hr} ; \mathrm{n}=7 ; \mathrm{p}<0.01$ vs controls) and $\mathrm{C} 3 \mathrm{H}(-1.52 \pm 0.2 \mathrm{hr} ; \mathrm{n}=6 ; \mathrm{p}<0.01$ vs controls) animals. C57BL mice showed almost similar phase delays at CT18 $(-1.71 \pm 0.5 \mathrm{hr} ; \mathrm{n}=6 ; \mathrm{p}<0.01$ vs controls) and at CT14, whereas in $\mathrm{C} 3 \mathrm{H}$ mice phase delays were significantly smaller at C T $18(-0.78 \pm 0.16 \mathrm{hr} ; n=6 ; p<0.05$ vs controls and vs C T 14) compared with that at CT14. The light-induced phase delays at C T18 were significantly larger $(p<0.01)$ in C57B L mice than in C3H mice (Fig. 2A).

\section{Induction of pCREB-IR in the SCN after light exposure}

Light pulses applied during subjective day (CT06, for $\mathrm{C} 3 \mathrm{H}, \mathrm{n}=$ 5; for C57B L, $n=3$; C T 10, for $\mathrm{C} 3 \mathrm{H}, \mathrm{n}=3$; for $\mathrm{C} 57 \mathrm{BL}, \mathrm{n}=4$ ) or handling the animals in $D D$ at any of the time points investigated (for both strains, $n=4$ ) did not induce pCREB-IR. In both mouse strains a light pulse during subjective night (CT14 and C T18, for both strains, $n=3$ for all groups) induced a robust pCREB-IR within the SCN (Figs. 2B, 3, 4). In the ventrolateral part of the $S C N$, double staining for cell nuclei showing a PCREB-IR with perikarya showing a V I P-IR could be observed. In addition, some SCN cells showed only PCREB-IR, whereas others showed only VIP-IR (Fig. 4). Thus, because the PCREB-IR overlaps only partially with the VIP-IR that marks cells receiving a direct retinal input (Tanaka et al., 1993), the phosphorylation of CREB may contribute to the integration of photic information with pacemaker adjustment.

\section{PACAP- and glutamate-induced PCREB-IR in the SCN in vitro}

There was no significant difference in the levels of basal PCREB-IR in untreated slices of both mouse strains, at any of the zeitgeber times investigated (controls, for both strains, $n=$ 16). Induction of $p C R E B-I R$ by the two agonists was phase- dependent, but with contrasting windows of sensitivity. G lutamate stimulated CREB phosphorylation at ZT 14 and ZT 18 in the SCN of C3H ( $Z$ T14, $n=5 ; p<0.001$ vs controls; ZT18, $n=5 ; p<0.05$ vs controls) and C57BL (ZT 14, $n=4 ; p<0.001$ vs controls; $Z T 18, n=8 ; p<0.05$ vs controls) mice. G lutamate application at Z T06 (for $\mathrm{C} 3 \mathrm{H}, \mathrm{n}=5$; for $\mathrm{C} 57 \mathrm{BL}, \mathrm{n}=7$ ) or ZT10 (for $\mathrm{C} 3 \mathrm{H}, \mathrm{n}=6$; for $\mathrm{C} 57 \mathrm{BL}, \mathrm{n}=7$ ) did not induce a significant $P C R E B-I R$ in either strain (Fig. 5). PACAP application at ZTO6 (for $\mathrm{C} 3 \mathrm{H}, \mathrm{n}=4$; for $\mathrm{C} 57 \mathrm{BL}, \mathrm{n}=3$ ), at ZT14 (for both strains, $n=4$ ), or at $Z T 18$ (for both strains, $n=3$ ) did not induce a $P C R E B-I R$ in the SC N of either mouse strain. In contrast, at ZT10 PACAP treatment evoked a robust PCREB-IR in the SCN of C3H and C57BL mice (for both strains, $n=5 ; p<0.001$ vs controls; Fig. 5). The PACA P- or glutamate-induced PCREB-IR did not differ between C $3 \mathrm{H}$ and C57BL mice at any of the time points investigated. In both strains the $P C R E B-I R$ was found predominantly in the ventrolateral region of the $\mathrm{SCN}$ (see Fig. 7).

\section{Effects of melatonin on PACAP- and glutamate- induced CREB phosphorylation}

Melatonin was applied to SCN slices at ZT10 and ZT14, because at these time points a maximal PCREB-IR was induced by PACA P and glutamate, respectively. A lthough melatonin (1 nM) alone was without effect (data not shown), it significantly inhibited the PACA P-induced CREB phosphorylation in C $3 H(n=4 ; p<0.001)$ and C57BL $(n=5 ; p<0.001)$. In contrast, the glutamate-evoked $\mathrm{PCREB}$-IR remained unaltered in both mouse strains when slices were incubated with melatonin before and during drug treatment (for $\mathrm{C} 3 \mathrm{H}, \mathrm{n}=5$; for C57BL, $n=4)$ (Figs. 6, 7).

\section{DISCUSSION}

A daptation of the circadian pacemaker in the mammalian SC N to changing environmental light/dark cycles is fundamental for sur- 

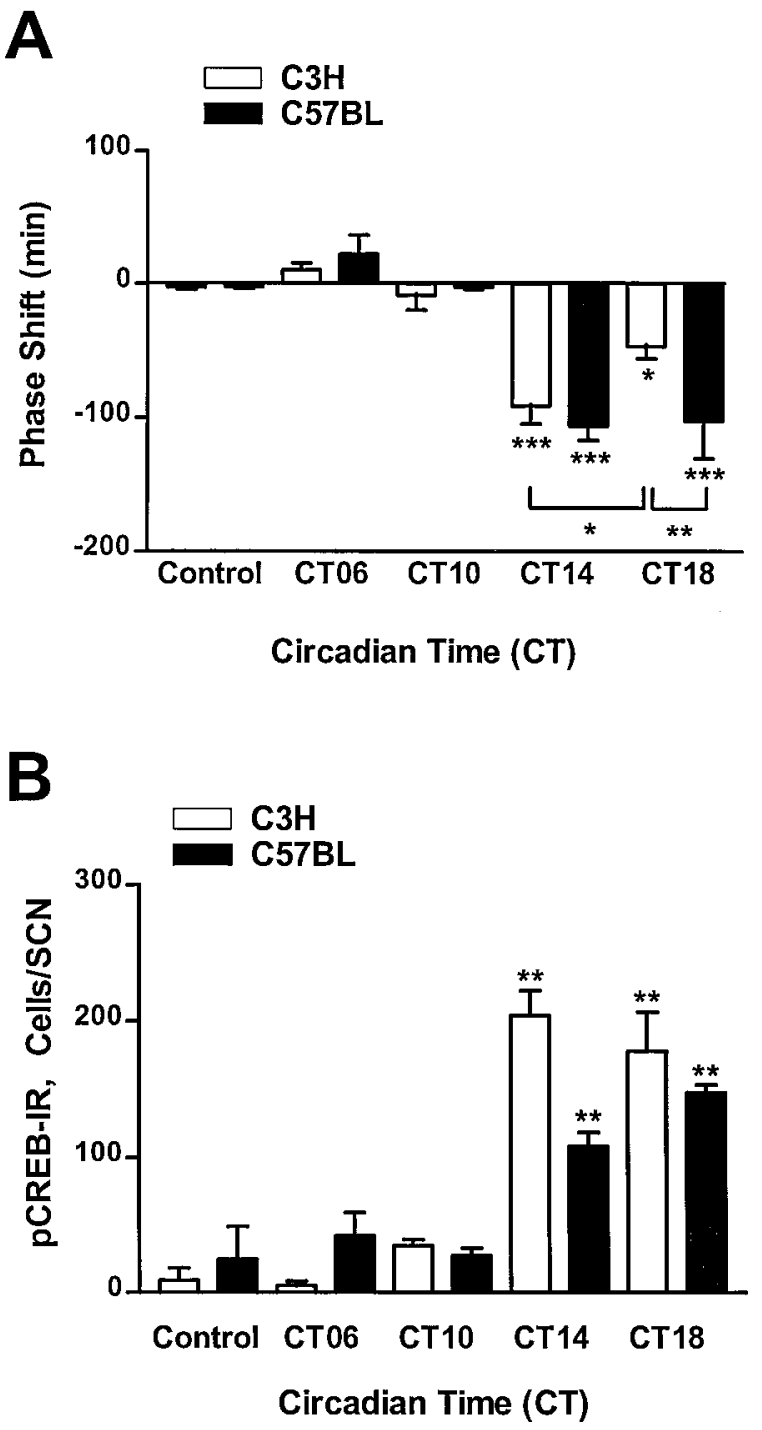

Figure 2. Comparison of light-induced effects on locomotor activity ( $A$ ) and phosphorylation of CREB (B) in the mouse SC N. C3H and C57BL mice were kept in constant darkness (dim red light), and brief light pulses ( $15 \mathrm{~min} ; 1000$ lux) were delivered at the times indicated. Control animals were handled but not exposed to light. A, Light-induced phase shifts in locomotor activity were analyzed from recorded actograms. Negative values represent phase delays; positive values represent phase advances. $N$ ote that the light exposure at C T 18 induced significantly smaller phase delays in $\mathrm{C} 3 \mathrm{H}$ mice compared with $\mathrm{C} 57 \mathrm{BL}$ mice. $\mathrm{B}$, Light-induced PCREB-IR (see also Fig. 3) was quantified in cryostat-cut serial brain sections of the hypothalamic region containing the SC N. E ach data point represents the mean \pm SEM of 5-16 (A) or 3-9 (B) experiments. $* p<$ $0.05 ; * * p<0.01 ; * * * p<0.001$.

vival. Our analyses of signaling events in the mouse SC N highlight a major role for the transcription factor CREB as a molecular interface between various resetting cues accessing the circadian clock. In particular, we demonstrate that light and two important transmitters of the RHT, PACA P and glutamate, activate CRE B in the SC N by phosphorylation during discrete time windows that match with the corresponding temporal domains for these signals to induce phase shifts in vivo (for review, see Gillette, 1996). The comparative analyses of melatonin-deficient and -proficient mice show that sensitivity windows for resetting cues are determined predominantly by cell-autonomous mechanisms within the SC N rather than by a phasic melatonin signal.

\section{$\mathrm{C} 3 \mathrm{H}$}

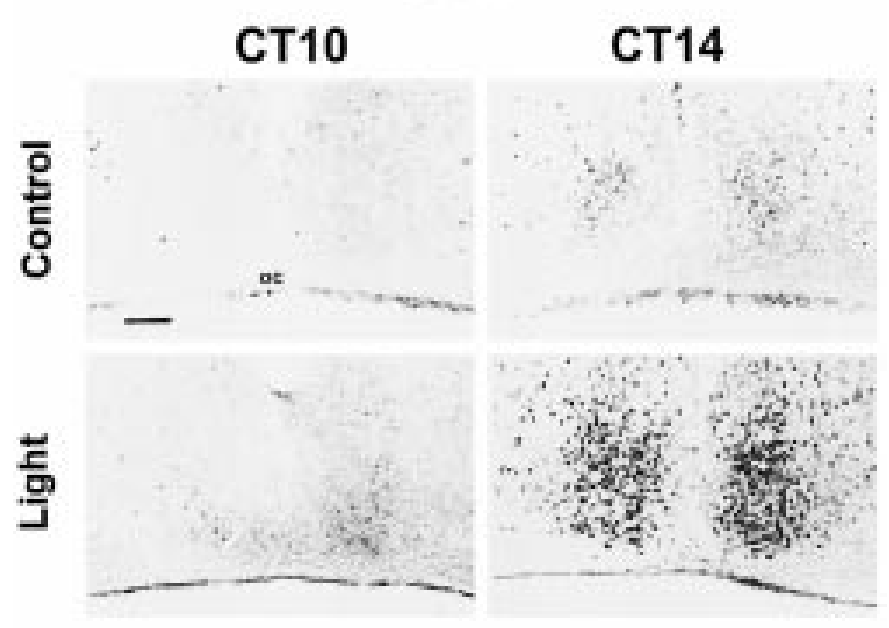

C57BL

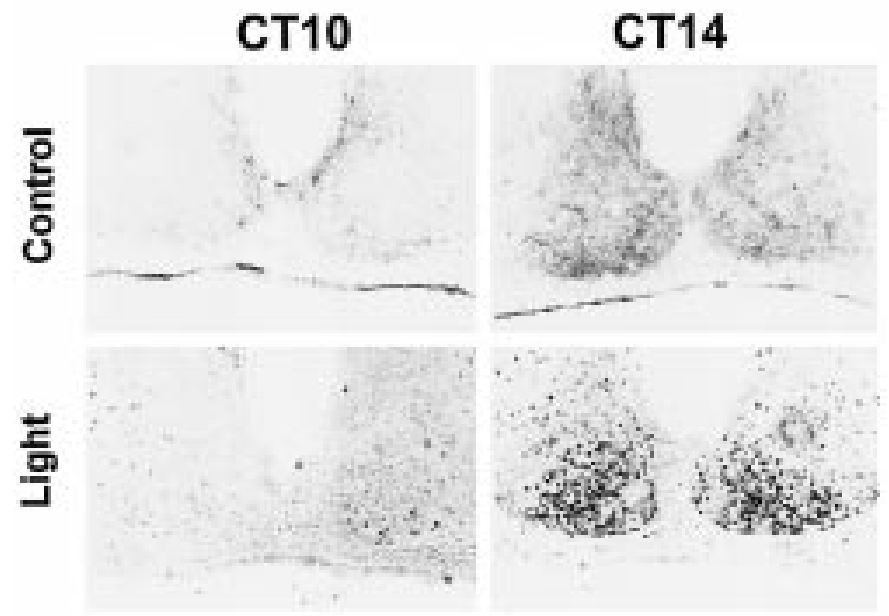

Figure 3. I mmunocytochemical demonstration of a light-induced CREB phosphorylation in mouse SCN. R epresentative coronal sections through the hypothalamic region containing the $\mathrm{SC} \mathrm{N}$ of $\mathrm{C} 3 \mathrm{H}$ and $\mathrm{C} 57 \mathrm{BL}$ mice are shown. Nuclear PCREB-IR in the SCN of mice under free-running conditions was induced when a brief light pulse ( $\mathrm{L}$ ight) was delivered $2 \mathrm{hr}$ after activity onset (C T 14) or in the middle of the subjective night (C T 18; data not shown). Light stimulation had no effect when given $2 \mathrm{hr}$ before activity onset (C T10) or in the middle of the subjective day (C T 06; data not shown). Control animals not exposed to the light stimulus (Control) showed only a weak basal PCREB-IR within the SC N. Scale bar, $50 \mu \mathrm{m}$. oc, O ptic chiasm.

The principal natural stimulus for the phase adjustment of the circadian oscillator is light (for review, see Meijer, 1991). The light-induced phase delays in locomotor activity of both mouse strains are consistent with previous reports (Schwartz and Z immerman, 1990; Benloucif and Dubocovich, 1996). Exogenous melatonin was reported to attenuate photically induced phase delays in C 3H mice at C T 14 and C T 18 (D ubocovich et al., 1996). Because $\mathrm{C} 3 \mathrm{H}$ mice show an elevated melatonin synthesis only during the second half of the night (Goto et al., 1989), the endogenous hormone effect on light-induced phase shifts should not be detectable at C T 14. I ndeed, phase delays are attenuated at CT18 as compared with CT14. Although this interpretation 

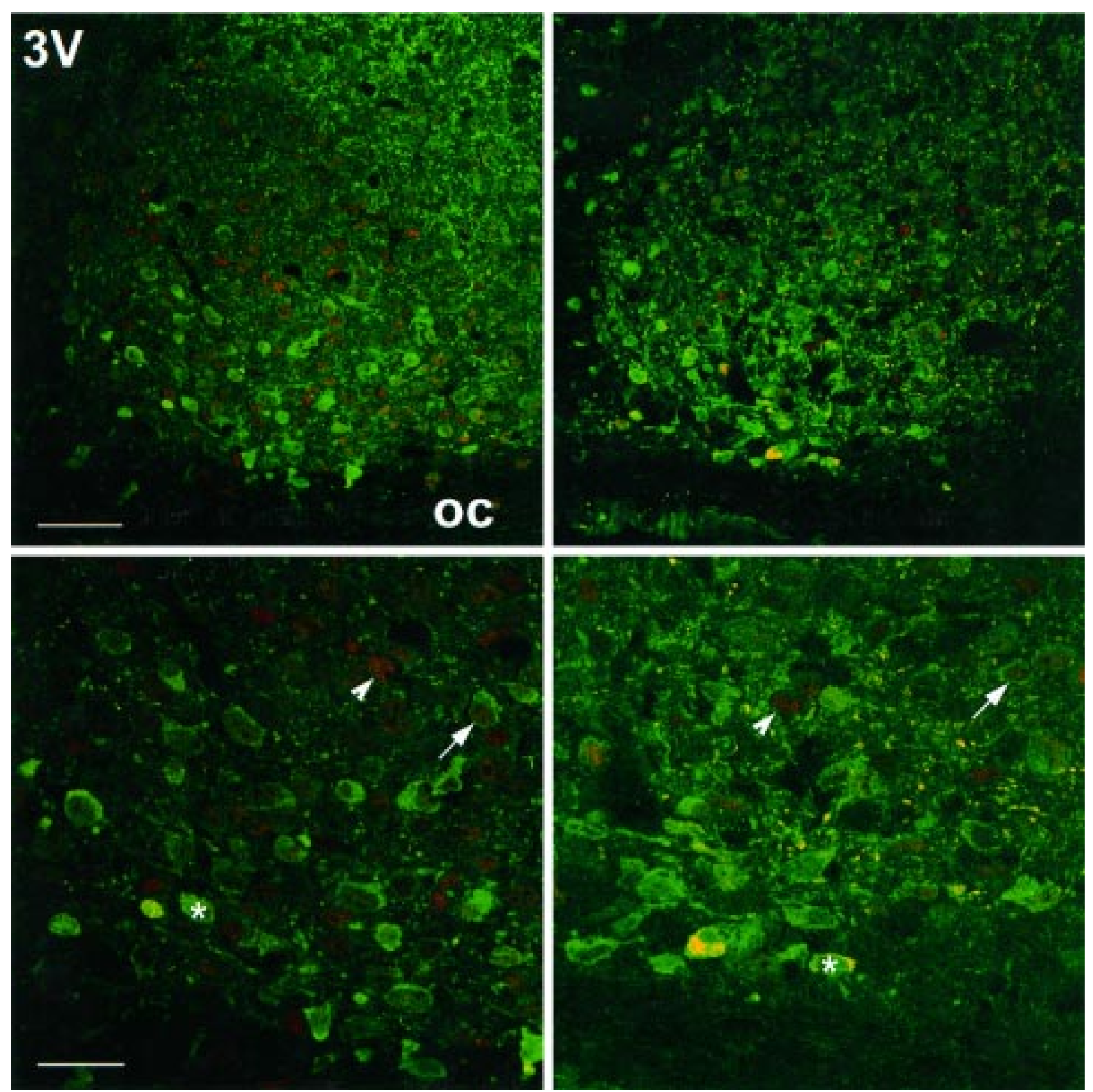

Figure 4. Distribution of PCREB-IR and VIP-IR in the mouse SC N. C3H (left) and C57BL (right) mice, kept under standard light/dark conditions, were exposed to bright white light (10 $\mathrm{min}$ ) at ZT 14. D ouble-label immunocytochemistry using a confocal laser-scanning microscope shows the spatial distribution of light-induced PCREB-IR nuclei (red) and V IP-IR cells ( green). Several cells show both nuclear pCRE B-IR and cytoplasmic V I P-IR (arrows); some cells show either a nuclear pCR EB-IR (arrowheads) or a cytoplasmic V I P-IR (asterisks) only. Scale bars: upper, $50 \mu \mathrm{m}$; lower, $25 \mu \mathrm{m}$. oc, O ptic chiasm; 3V, third ventricle.

awaits further experimentation, it is supported by consistently large phase delays in melatonin-deficient C57BL mice at C T14 and at C T18 (E bihara et al., 1986; Goto et al., 1989). In both mouse strains, resetting light pulses were always associated with CREB phosphorylation in the SCN, a molecular link originally observed in the Syrian hamster ( $G$ inty et al., 1993). N otably, the dynamic profile of the $P C R E B-I R$ was not different between the two mouse strains and, thus, independent of the size of the phase delays. These results imply that endogenous melatonin may modulate the magnitude of light-induced phase shifts, but not the acute cellular responses of the SC N to light, including the induc- tion of $\mathrm{PCREB}$. These observations profile a feedback function for the pineal hormone, with melatonin potentially setting the gain of SC N sensitivity to resetting stimuli on a diurnal and a seasonal basis.

Glutamate, a principal transmitter of the R H T (for review, see E bling, 1996), induced CRE B phosphorylation in the SC N probably via activation of the $\mathrm{Ca}^{2+} /$ calmodulin-signaling pathway. In both mouse strains glutamate-induced CREB phosphorylation was observed during subjective night but not during subjective daytime. This observation extends the results of a previous study in rat, which compared the effects of glutamate at C T07 and 

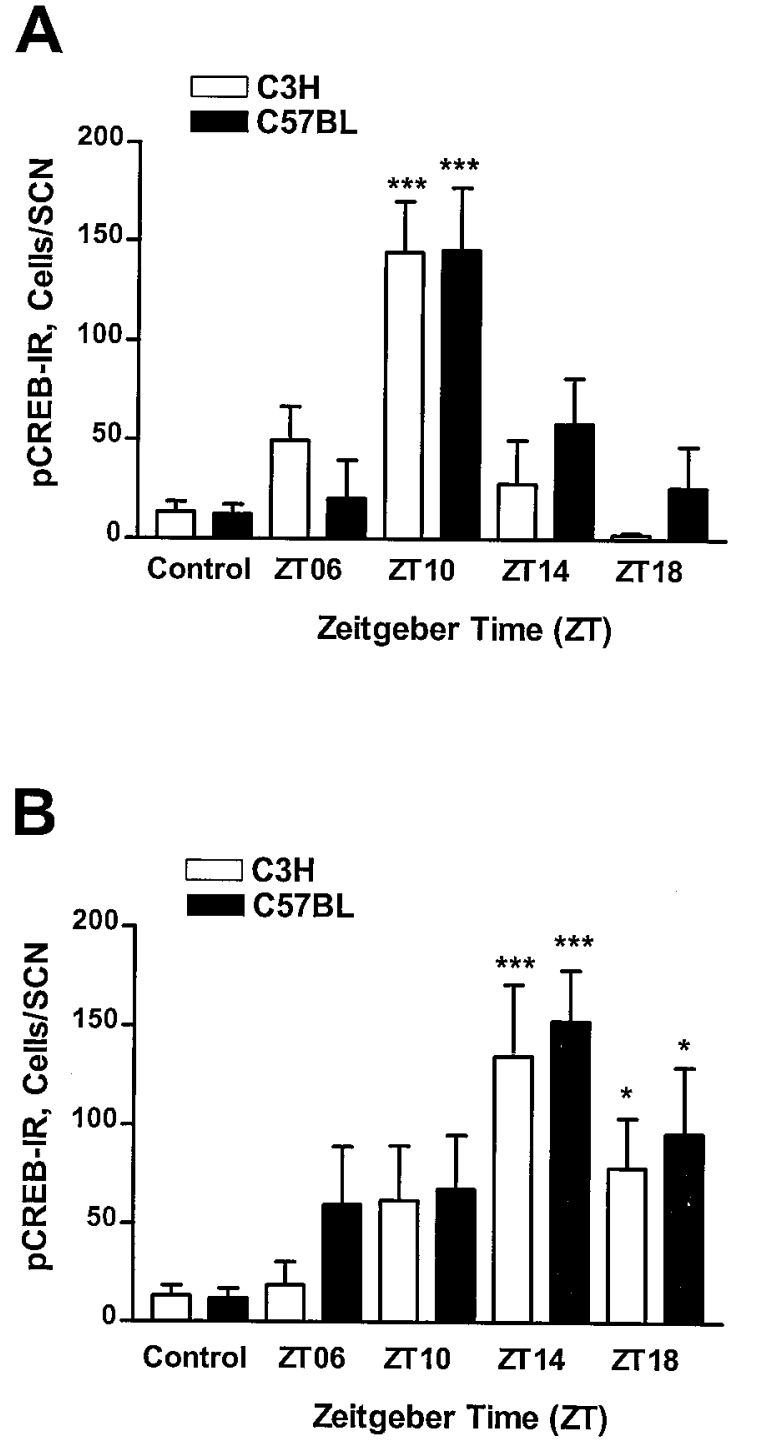

Figure 5. Semiquantitative analysis of the PCREB-IR induced by PACA P (A) or glutamate (B) in mouse SCN brain slices. A, PACA P application $(100 \mathrm{~nm})$ evoked a robust pCREB-IR in the SCN of C $3 \mathrm{H}$ and C57BL mice when applied at ZT10 but had no effect at ZT14, ZT18, or ZT06. B, Glutamate application $(100 \mu \mathrm{M})$ induced pCREB-IR in the SCN of C3H and C57B L mice at Z T 14 and Z T 18 but had only slight effects at Z T 06 and Z T 10. U ntreated slices (controls) showed a very low basal pCR E B-IR (see also Fig. 7). Each data point represents the mean \pm SEM of four to nine animals. Asterisks indicate significantly different values of stimulated slices compared with controls; *p $<0.05 ; * * * p<0.001$.

C T20 (Ding et al., 1997). The temporal window of sensitivity to glutamate coincides with the period of glutamate-induced phase shifts in rat SCN explants (Ding et al., 1994). Notably, CREB phosphorylation is a consistent cellular response to resetting glutamatergic cues, regardless of the direction of the phase response, because it can be induced at early subjective night (this study) when glutamate induces maximal phase delays (Ding et al., 1994) and at C T20 (Ding et al., 1997) when glutamate induces phase advances (Ding et al., 1994).

PACA P is an RH T transmitter that stimulates CA M P production in the SC N via the PACA P-R 1 receptor ( $H$ annibal et al., 1997). Because PACA P levels in the rat SC N are reduced by light (Fukuhara et al., 1997), any release of PA CA P by R H T
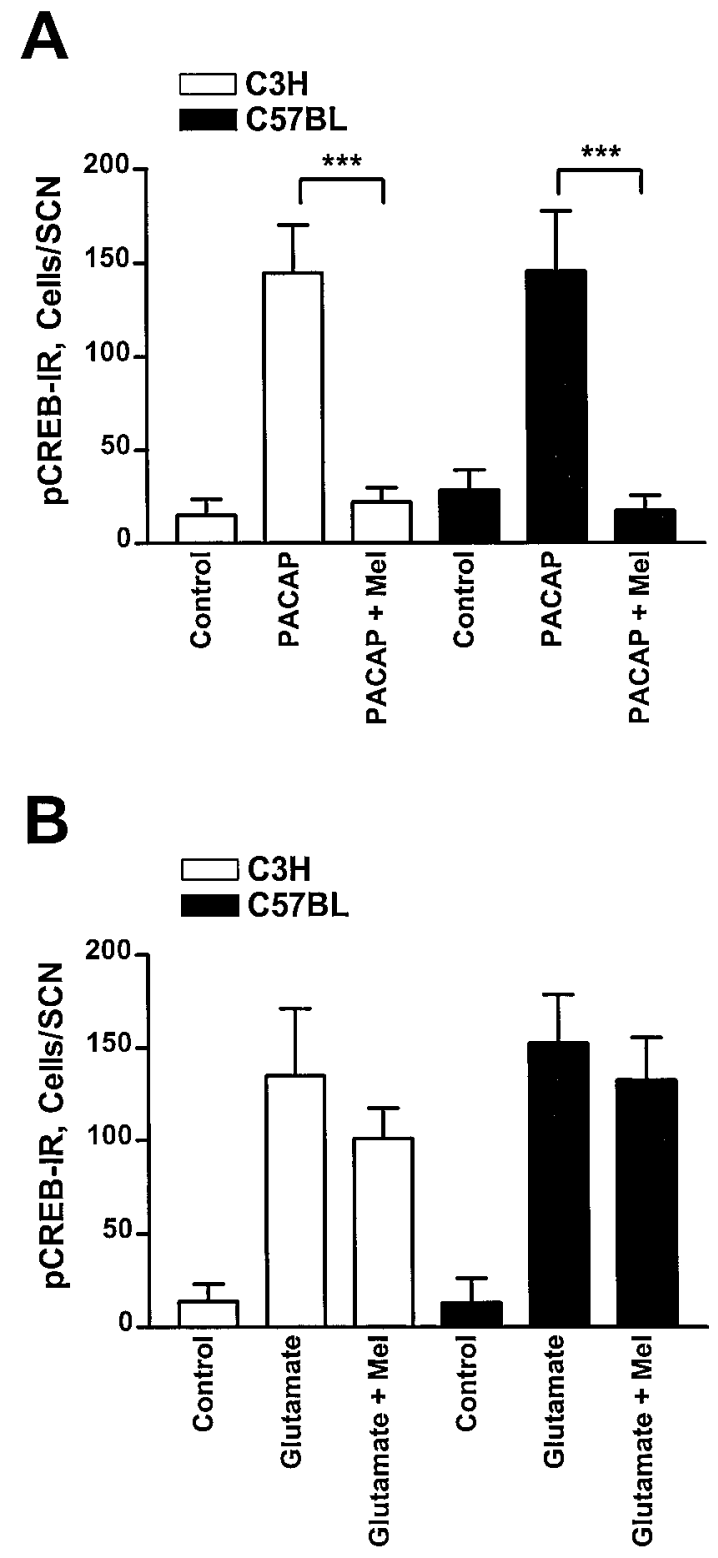

Figure 6. Semiquantitative analysis of the effects of melatonin on the PACA P- or the glutamate-induced PCREB-IR. A, A preceding incubation of $\mathrm{SC} \mathrm{N}$ brain slices with melatonin ( $\mathrm{Mel} ; 1 \mathrm{nM}$ ) prevented the induction of a PCRE B-IR by PACA P at Z T 10 in both mouse strains. B, The glutamateinduced $P C R E B-I R$ in the SCN of both mouse strains at ZT14 was unaltered when slices were preincubated with melatonin. Each data point represents the mean \pm SEM of four to eight animals; ***p $<0.001$.

fibers would make the SCN "sense darkness" and allow the pacemaker to readjust rapidly to ambient lighting conditions. In our hands PA CA P induced a maximal CREB phosphorylation at Z T10, and the effect was not different between the two mouse strains. Therefore, as for glutamate and light, the gate of sensitivity is set independently of any rhythmic melatonin signal from the pineal gland. The sensitivity window for PA CA P overlaps temporally with phase advances induced either by dark pulses in vivo (E llis et al., 1982) or by application of CA M P analogs in vitro (Prosser and Gillette, 1989). The phasic induction of $\mathrm{PCREB-IR}$ by PACA P described here for mice conforms to studies in rat showing that the resetting 


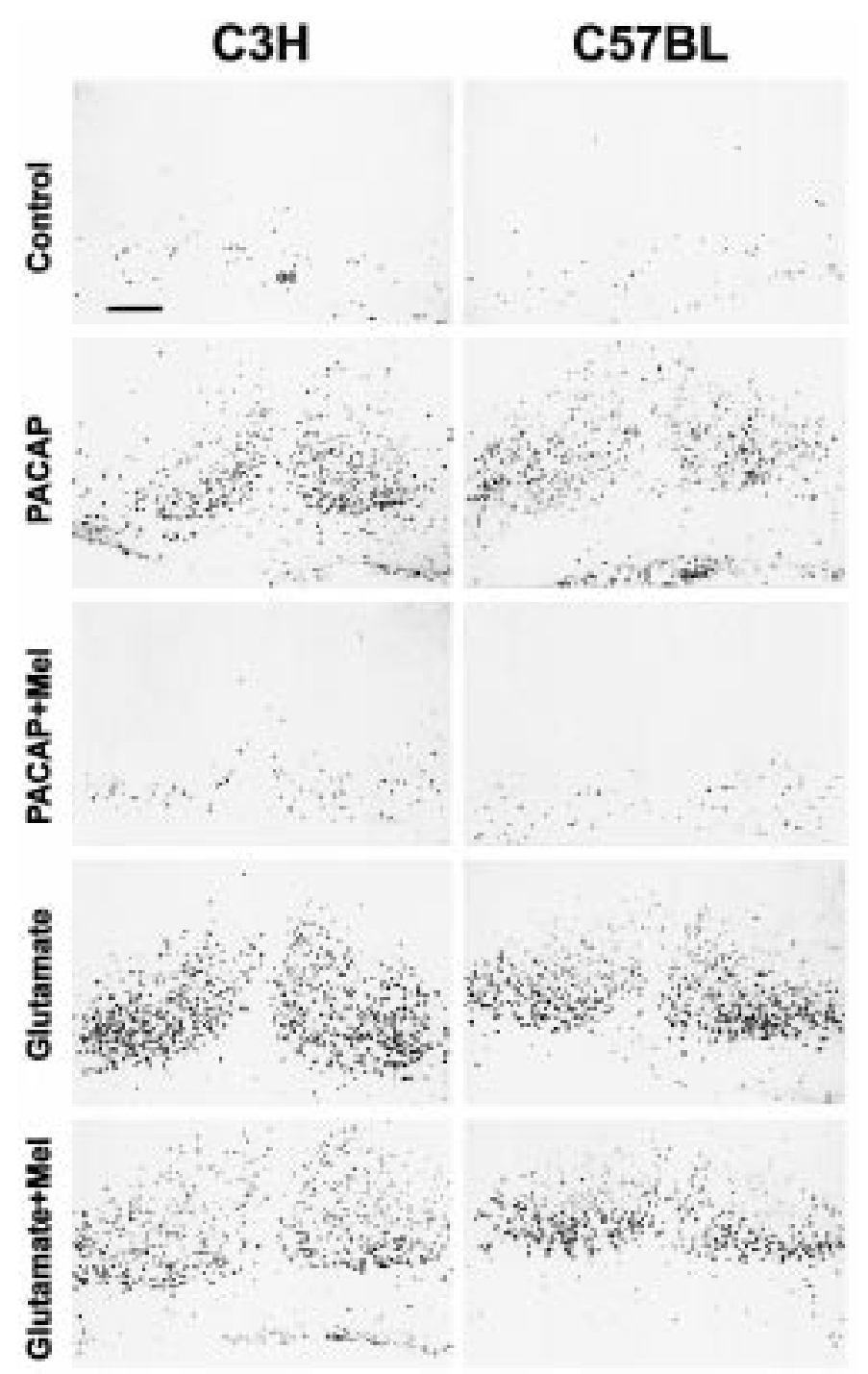

Figure 7. E ffects of melatonin on the PACA P- or the glutamate-induced PCREB-IR. Untreated control slices (Control) showed no basal PCREB-IR in the SC N of both mouse strains after $2 \mathrm{hr}$ in culture (Z T 10) or after $6 \mathrm{hr}$ in culture (ZT14; data not shown). PACA P application (PACAP) at ZT10 induced a nuclear PCREB-IR in the SC N of C3H and C57BL mice. The PACA P effect was suppressed when slices were preincubated with melatonin (PACAP $+\mathrm{Mel}$ ). Glutamatergic stimulation (G lutamate) of slices at ZT14 evoked a nuclear pCR E B-IR in the SC N of both mouse strains. Glutamate-effects were not affected by melatonin ( $\mathrm{Glutamate}+\mathrm{M} \mathrm{el}$ ). I $\mathrm{n}$ both mouse strains melatonin alone did not induce a pCREB-IR in the SCN (data not shown). Scale bar, $50 \mu \mathrm{m}$. oc, O ptic chiasm.

action of this peptide is restricted to subjective daytime ( $\mathrm{H}$ annibal et al., 1997). However, Hannibal et al. investigated the resetting action of PACA P at ZTO6 and not at ZT10, a time point when we observed maximal induction of $P C R E B$ in mice. A II data support the concept that phosphorylation of CREB mediates PA CA P-mediated retinal signaling to the clock.

Our investigations with the two RHT transmitters imply a switch in SC N sensitivity between PA CA P (daytime), activating the CA M P/adenylate cyclase-signaling pathway (Gillette, 1996), and glutamate (nighttime), stimulating the $\mathrm{Ca}^{2+} /$ calmodulinsignaling pathway (Ebling, 1996). This switch occurs endogenously around the light/dark transition and is set independently of a phasic melatonin signal, because it is present in $\mathrm{C} 3 \mathrm{H}$ and also in C57BL mice. A II these findings support the notion that transcription is part of the mechanism for adjustment of oscillator timing.

Exogenous melatonin applied around dusk is also a potent resetting cue in rats (A rmstrong et al., 1986), in Siberian hamsters (D uffield et al., 1999), and in some strains of mouse (Benloucif and D ubocovich, 1996). In the current study, melatonin reversed PACAP-induced phosphorylation of CREB when applied at ZT10 in both $\mathrm{C} 3 \mathrm{H}$ and C57B L mice. This conforms to an autoradiographic demonstration of melatonin-binding sites in the SC N of both strains (Siuciak et al., 1990). C haracterization of the two melatonin receptor subtypes expressed in the mouse $\mathrm{SCN}$, the $\mathrm{M} \mathrm{el}_{1 a}$ (Roca et al., 1996) and the $\mathrm{M} \mathrm{el}_{1 b}$ receptor (Liu et al., 1997), has shown that both are coupled to inhibition of adenylate cyclase activity (for review, see R eppert et al., 1996). It is therefore likely that in the SC N of both mouse strains, molecular cross talk exists between melatonin- and PA CA P-regulated signaling at the level of CA M P accumulation but not between melatonin and the glutamate-activated $\mathrm{Ca}^{2+}$-dependent pathway. The insensitivity of this signal transduction pathway toward melatonin may secure the responsiveness of the SCN to light-induced phase shifts at night. This is consistent with a recent report that melatonin cannot affect glutamatergic induction of $P C R E B-I R$ in the SC N of neonatal Syrian hamsters (M CNulty et al., 1998).

O ur data suggest that CREB serves in the mouse SCN as a molecular interface to translate a gated transmitter preference for adjustment of the phase of the pacemaker. This convergence of multiple and separately inducible signaling pathways onto CREB phosphorylation is well known (M ontminy et al., 1990; Dash et al., 1991; Sheng et al., 1991). In the mouse SC N such convergence seems to be achieved via a rapid and efficient but restricted intracellular molecular cross talk and may affect and adjust clockwork transcription. The evidence supporting this idea can be derived from observations that in the SC N of both mouse strains, CREB phosphorylation is induced within a few minutes by (1) light stimuli that reset the SCN pacemaker, (2) glutamatergic receptor activation, and (3) PACA Pergic receptor activation. Importantly, (4) melatonin interferes selectively with the PACA P-induced CREB phosphorylation in both mouse strains. The case for granting CREB a central role for the integration of photic information into the clockwork can also be inferred from known molecular details of this transcription factor ( $G$ inty et al., 1993; M cN ulty et al., 1994, 1998; T amotsu et al., 1995; K ako et al., 1996). It should be noted, however, that our data do not allow us to eliminate a possible parallel processing of clock resetting and CREB phosphorylation, both affected by PACA P and glutamate signaling (Gillette, 1996).

The very rapid stimulus-induced CREB phosphorylation in the rodent $\mathrm{SCN}$ allows this event to intersect with clock mechanisms at the earliest time point possible. CREB phosphorylation induced by photic stimulation at nighttime occurs before the transcriptional induction of immediate early genes that is associated with light-induced phase shifts in clock function (Wollnik et al., 1995; for review, see $\mathrm{H}$ astings, 1997) and before the rapid elevation of mPerl and mPerlI mRNA levels, the putative mouse ortholog of the Drosophila clock gene period (Shearman et al., 1997; Zylka et al., 1998). A s CREB phosphorylation is known to be a key step in coupling shortterm neuronal stimuli to long-term intracellular responses (Y amamoto et al., 1988; Montminy et al., 1990), the lightinduced CREB phosphorylation in rodent SCN may be the 
molecular initiator to reset the phase of circadian behavioral cycles. It may be envisioned that pCREB induction affects clock genes like mPer to cause phase delays during the early night by retarding the spontaneous decline in SC N activity and to cause phase advances during late night by activating a precocious increase in the molecular oscillation, based on mPer.

\section{REFERENCES}

A rmstrong SM, C assone V M, Chesworth MJ, Redman JR, Short RV (1986) Synchronization of mammalian circadian rhythms by melatonin. J N eural Transm 21:375-394.

Benloucif S, D ubocovich ML (1996) Melatonin and light induce phase shifts of circadian activity rhythms in the $\mathrm{C} 3 \mathrm{H} / \mathrm{HeN}$ mouse. J Biol R hythms 11:113-125.

Cassone V M , R oberts M H, M oore RY (1987) M elatonin inhibits metabolic activity in the rat suprachiasmatic nuclei. Neurosci Lett 81:29-34.

C astel M, Belenky M, Cohen S, Ottersen OP, Storm-M athisen J (1993) Glutamate-like immunoreactivity in retinal terminals of the mouse suprachiasmatic nucleus. Eur J Neurosci 5:368-381.

D ash PK, Karl K A, Colicos M A, Prywes R, K andel ER (1991) CA M P response element-binding protein is activated by $\mathrm{C}^{2+} /$ calmodulin- as well as CAM P-dependent protein kinase. Proc Natl Acad Sci USA 88:5061-5065.

Ding J M , Chen D, Weber ET, Faiman LE, R ea M A , G illette M U (1994) $R$ esetting the biological clock: mediation of nocturnal circadian shifts by glutamate and NO. Science 266:1713-1717.

Ding J M, Faiman LE, H urst WJ, Kuriashkina LR, Gillette M U (1997) $R$ esetting the biological clock: mediation of nocturnal CRE B phosphorylation via light, glutamate, and nitric oxide. J Neurosci 17:667- 675.

Ding J M, Buchanan GF, Tischkau SA, Chen D, Kuriashkina L, Faiman LE, A Ister J M, M cPherson PS, C ampbell K P, G illette M U (1998) A neuronal ryanodine receptor mediates light-induced phase delays of the circadian clock. Nature 394:381-384.

D ubocovich M L, Benloucif S, M asana M I (1996) M elatonin receptors in the suprachiasmatic nucleus. Behav Brain R es 73:141-147.

D uffield GE, Hastings M H, Ebling FJ P (1999) Investigations into the regulation of the circadian system by dopamine and melatonin in the adult Siberian hamster (Phodopus sungorus). J Neuroendocrinol, in press.

E bihara S, M arks T, H udson DJ , M enaker M (1986) Genetic control of melatonin synthesis in the pineal gland of the mouse. Science 231:491- 493.

E bling FJ P (1996) The role of glutamate in the photic regulation of the suprachiasmatic nucleus. Prog Neurobiol 50:109-132.

Ellis G B, M cK leen RE, T urek F W (1982) D ark pulses affect the circadian rhythm of activity in hamsters kept in constant light. A $\mathrm{m}$ J Physiol 242:44-50.

Fukuhara C, Suzuki N, M atsumoto $Y$, Nakayama $Y$, A oki K, T sujimoto G, Inouye SI T, M asuo Y (1997) Day-night variation of pituitary adenylate cyclase-activating polypeptide (PACA P) level in the rat suprachiasmatic nucleus. N eurosci L ett 229:49-52.

Gillette MU (1986) The suprachiasmatic nuclei: circadian phase-shifts induced at the time of hypothalamic slice preparation are preserved in vitro. Brain Res 379:176-181.

Gillette M U (1996) Regulation of entrainment pathways by the suprachiasmatic circadian clock: sensitivities to second messengers. In: Progress in brain research, $\mathrm{V}$ ol III, $\mathrm{H}$ ypothalamic integration of circadian rhythms (Buijs RM, Kalsbeek A, Romijn HJ, Pennartz CM A, M irmiran M, eds), pp 121-132. A msterdam: Elsevier.

Ginty DD, K ornhauser J M, Thompson M A, Bading $H$, M ayo KE, Takahashi J S, G reenberg M E (1993) R egulation of CREB phosphorylation in the suprachiasmatic nucleus by light and a circadian clock. Science 260:238-241.

G oto M, O shima I, Tomita T, E bihara S (1989) M elatonin content of the pineal glands in different mouse strains. J Pineal Res 7:195-204.

$H$ annibal J, Ding J M, Chen D, Fahrenkrug J, Larsen PJ, Gillette M U, Mikkelsen JD (1997) Pituitary adenylate cyclase-activating peptide (PACA P) in the retinohypothalamic tract: a potential daytime regulator of the biological clock. J Neurosci 17:2637-2644.

$\mathrm{H}$ astings M H (1997) C entral clocking. Trends N eurosci 20:459- 463.

K ako K, Wakamatsu H, I shida N (1996) c-fos CRE-binding activity of CRE B/A TF family in the SC N is regulated by light but not a circadian clock. N eurosci L ett 216:159-162.
K lein DC, M oore RY, R eppert SM (1991) Suprachiasmatic nucleus: the mind's clock (K lein DC, M oore R H, R eppert SM, eds), pp 1- 467. N ew Y ork: Oxford UP.

Kopp M, M eissl H, Korf H W (1997) The pituitary adenylate cyclaseactivating polypeptide-induced phosphorylation of the transcription factor CREB (CAM P response element binding protein) in the rat suprachiasmatic nucleus is inhibited by melatonin. Neurosci Lett 227:145-148.

K ornhauser J M, Nelson DE, M ayo K E, Takahashi JS (1990) Photic and circadian regulation of c-fos gene expression in the hamster suprachiasmatic nucleus. Neuron 5:127-134.

Liu C, Weaver DR, J in X, Shearman LP, Pieschl RL, Gribkoff VK, Reppert SM (1997) Molecular dissection of two distinct actions of melatonin on the suprachiasmatic circadian clock. N euron 19:91-102.

M cA rthur A J, Gillette M U, Prosser R A (1991) M elatonin directly resets the rat suprachiasmatic circadian clock in vitro. Brain Res 565:158-161.

M cNulty S, R oss AW, Barrett B, H astings M H, M organ PJ (1994) M elatonin regulates the phosphorylation of $C R E B$ in ovine pars tuberalis. J Neuroendocrinol 6:523-532.

M cNulty S, Schurov IL, Sloper PJ, H astings M H (1998) Stimuli which entrain the circadian clock of the neonatal Syrian hamster in vivo regulate the phosphorylation of the transcription factor CREB in the suprachiasmatic nucleus in vitro. Eur J Neurosci 10:1063-1072.

M eijer JH (1991) Integration of visual information by the suprachiasmatic nucleus. In: Suprachiasmatic nucleus: the mind's clock (K lein DC, Moore RH, R eppert SM, eds), pp 107-119. N ew Y ork: Oxford UP.

Montminy M R, Gonzalez GA, Y amamoto K K (1990) Regulation of CA M P-inducible genes by CREB. Trends Neurosci 13:184-188.

Pfeffer M, Kühn R, K rug L, K orf H W, Stehle J H (1998) R hythmic variation in $\beta 1$-adrenergic receptor $\mathrm{mR} \mathrm{NA}$ levels in rat pineal gland: circadian and developmental regulation. Eur J Neurosci 10:2896-2904.

Prosser R A, Gillette M U (1989) The mammalian circadian clock in the suprachiasmatic nuclei is reset in vitro by CAMP. J Neurosci 9:1073-1081.

Prosser RA, M cA rthur A J, Gillette MU (1989) cGM P induces phase shifts of a mammalian circadian pacemaker at night, in antiphase to CA M P effects. Proc Natl A cad Sci U SA 86:6812-6815.

R edman J, A rmstrong SM, N g K T (1983) Free-running activity rhythms in the rat: entrainment by melatonin. Science 219:1089-1091.

Reppert SM, Weaver DR, Godson C (1996) M elatonin receptors step into the light: cloning and classification of subtypes. Trends Pharmacol Sci 17:100-102.

R oca A L, Godson C, Weaver DR, R eppert SM (1996) Structure, characterization, and expression of the gene encoding the mouse $\mathrm{M} \mathrm{el}_{1 \mathrm{a}}$ receptor. Endocrinology 137:3469-3477.

Rusak B, Robertson HA, Wisden W, H unt SP (1990) Light pulses that shift rhythms induce gene expression in the suprachiasmatic nucleus. Science 248:1237-1240.

Schwartz W J Z immerman P (1990) C ircadian timekeeping in BA L B/C and C57BL /6 inbred mouse strains. J Neurosci 10:3685-3694.

Shearman LP, Zylka MJ, Weaver DR, K olakowski LF, R eppert SM (1997) T wo period homologs: circadian expression and photic regulation in the suprachiasmatic nuclei. Neuron 19:1261-1269.

Sheng M, Thompson M A, Greenberg ME (1991) CREB: a Ca ${ }^{2+}$ regulated transcription factor phosphorylated by calmodulindependent kinases. Science 252:1427-1430.

Shirakawa T, M oore RY (1994) G lutamate shifts the phase of the circadian neuronal firing rhythm in the rat suprachiasmatic nucleus. N eurosci L ett 178:47-50.

Siuciak J A, Fang J M, D ubocovich ML (1990) A utoradiographic localization of $2-\left[{ }^{125} \mathrm{I}\right]$ iodomelatonin binding sites in the brains of $\mathrm{C} 3 \mathrm{H} / \mathrm{HeN}$ and $\mathrm{C} 57 \mathrm{BL} / 6 \mathrm{~J}$ strains of mice. Eur J Pharmacol 180:387-390.

Stehle J H, Vanecek J, Vollrath L (1989) Effects of melatonin on spontaneous electrical activity of neurons in rat suprachiasmatic nuclei: an in vitro iontophoretic study. J N eural Transm 78:173-177.

Stehle J H, Pfeffer M, Kühn R, K orf H -W (1996) Light-induced expression of transcription factor ICER (inducible CA M P early repressor) in rat suprachiasmatic nucleus is phase-restricted. Neurosci Lett 217:169-172.

Sumova A, Ebling FJ P, M aywood ES, Herbert J, Hastings M H (1994) Non-photic circadian entrainment in the Syrian hamster is not associ- 
ated with phosphorylation of the transcriptional regulator CREB within the suprachiasmatic nucleus, but is associated with adrenocortical activation. Neuroendocrinology 59:579-589.

Tamotsu S, Schomerus C, Stehle J H, R oseboom PH, Korf H W (1995) Norepinephrine-induced phosphorylation of the transcription factor CRE B in isolated rat pinealocytes: an immunocytochemical study. C ell Tissue R es 282:219-226.

Tanaka M, I chitani Y, O kamura H, Tanaka Y , I bata Y (1993) The direct retinal projection to V I P neuronal elements in the rat SC N. B rain R es Bull 31:637-640.

Wollnik F, Brysch W, U hlmann E, Gillardon F, Bravo R, Z immermann $\mathrm{M}$, Schlingensiepen $\mathrm{KH}, \mathrm{H}$ erdegen $\mathrm{T}$ (1995) Block of c-F os and JunB expression by antisense oligonucleotides inhibits light- induced phase shifts of the mammalian circadian clock. Eur J Neurosci 7:388-393.

Y amamoto K K, Gonzalez GA, Biggs III W H, Montminy M R (1988) Phosphorylation-induced binding and transcriptional efficacy of nuclear factor CREB. Nature 334:494- 498.

Z hang Y, Takahashi J S, T urek F W (1996) Critical period for cycloheximide blockade of light-induced phase advances of the circadian locomotor activity rhythm in golden hamsters. Brain Res 740: 285-290.

Zylka M J, Shearman L P, W eaver DR, R eppert SM (1998) Three period homologs in mammals: differential light responses in the suprachiasmatic circadian clock and oscillating transcripts outside the brain Neuron 20:1103-1110. 